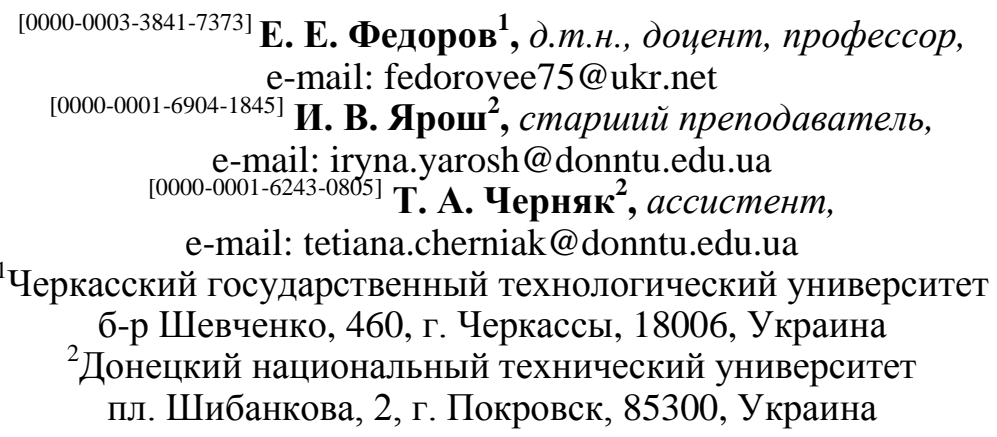

\title{
НЕЙРОСЕТЬ ТDNN ДЛЯ ДИАГНОСТИКИ СОСТОЯНИЯ ВЕНТИЛЯТОРНОЙ УСТАНОВКИ ГЛАВНОГО ПРОВЕТРИВАНИЯ
}

В статье рассмотрены и проанализированы существующие методы диагностики шахтного вентилятора. Исходя из выделенных основных преимуществ и недостатков указанных методов, разработан и реализован нейросетевой метод диагностики состояния вентиляторной установки главного проветривания. В основу данного метода заложена предложенная нейронная сеть TDNN, архитектура которой определена на основе проведенных экспериментальных исследований. Для ускорения процесса обучения авторской нейронной сети предложен пакетный режим обучения. Для оценки эффективности предложенного метода диагностики состояния вентиляторной установки главного проветривания была проведена серия численных исследований, результаты которых доказывают эффективность предложенной авторами нейронной сети и ее архитектуры.

Ключевые слова: диагностика, вентиляторная установка главного проветривания, нейронная сеть, TDNN, производственная безопасность, пакетный режим обучения.

У статті розглянуто та проаналізовано існуючі методи діагностики шахтного вентилятора. Виходячи з виділених основних переваг і недоліків зазначених методів, розроблено та реалізовано нейромережевий метод діагностики стану вентиляторної установки головного провітрювання. В основу иього методу закладено запропоновану нейронну мережу TDNN, що пов'язано з забезпеченням нею найліпшої точності діагностики. В роботі була визначена структура моделі нейромережі, обрана та обтрунтована ознака для здійснення оцінки ї̈ ефективності, виконане навчання з використанням редукиії при додаванні за навчальною множиною. Для прискорення процесу навчання авторської нейронної мережі запропоновано пакетний режим навчання; необхідно відмітити збільшення швидкості прямого і зворотного ходу при його використанні. Архітектура вказаної діагностичної моделі визначена на основі проведених експериментальних досліджень. Було виявлено, що збільшення кількості модулів вхідного шару нейромережі супроводжусться зменшенням значення середньоквадратичної помилки діагностики. Отримані результати свідчать про недоцільність використання понад шістнадияти модулів у вхідному шарі мережі. При наявності більшої кількості модулів буде спостерігатися досить незначна зміна значення отриманої помилки. Адекватність наведеної моделі характеризується вибором значень параметрів, що забезпечують мінімізацію показника середньоквадратичної помилки - мінімізацію різниці між виходом моделі і бажаним виходом. Обрана авторами нейромережа з часовою затримкою дозволяе досягти діагностичний результат з найменшим відхиленням і забезпечити підвищення якості результатів процесу діагностики вентилятора. Запропонований підхід може бути застосований в різних інтелектуальних системах, щзо забезпечують діагностичні процеси різного характеру.

Ключові слова: діагностика, вентиляторна установка головного провітрювання, нейронна мережа, TDNN, виробнича безпека, пакетний режим навчання.

Постановка проблемы. Одной из важнейших проблем, которые существуют сегодня в горнодобывающей промышленности, является повышение безопасности отрасли. Проблема аварийного оснащения горной техники вызвана стремительным увеличением доли истощения ее физических ресурсов. На сегодняшний день 
новейшие компьютерные диагностические системы [1-6] не обеспечивают своевременную и комплексную диагностику с одновременным учетом нескольких параметров. Это приводит к тому, что меры, направленные на предотвращение или уменьшение последствий аварий, могут быть приняты слишком поздно или без учета всех необходимых аспектов и факторов. Среди имеющегося оборудования шахты одну из важнейших ролей играют вентиляторные установки главного проветривания (ВУГП) [7-8], обеспечивающие нормальную жизнедеятельность персонала шахты. Именно поэтому немаловажной и актуальной является деятельность, посвященная разработке и всецелому исследованию интеллектуальных методов диагностики ВУГП.

Цель исследования заключается в разработке методики углубленного изучения процесса изменения состояния ВУГП.

Задачи исследования, поставленные и решенные для достижения цели:

- проанализировать существующие методы диагностики;

- разработать

искусственную нейронную сеть для диагностики; определить структуры ее модели;

- выбрать критерий оценки эффективности нейросетевой диагностической модели;

- выполнить обучение модели искусственной нейронной сети;

- провести численные исследования.

Изложение основного материала. До нынешних дней предложено достаточное число способов и подходов как средств для вибродиагностики. Следует выделить методы вычисления: пикового коэффициента; эксцесса; среднеквадратичного значения; огибающего спектра. Работа [1] содержит результаты сравнения характеристик приведенных методов, основываясь на которых огибающий спектральный метод признан как наиболее точный.

Использование нейронных сетей во время выполнения диагностики дает ощутимые преимущества:

- с использованием готовых моделей анализируются и изучаются взаимосвязи между факторами;

- отсутствует потребность в предположениях относительно распределения имеющихся факторов;

- предварительная информация факторах может отсутствовать;

(C) Е. Е. Федоров, И. В. Ярош, Т. А. Черняк, 2019 DOI: $10.24025 / 2306-4412.4 .2019 .184525$
- исходные данные могут быть в сильной степени коррелированными, неполными или зашумленными;

- возможно прослеживание высокой степени нелинейности во время анализа;

- прослеживается быстрое построение модели; наблюдается высокая адаптивность;

- возможна ситуация проведения многофакторного анализа системы;

- отсутствует необходимость в полном переборе всех возможных моделей;

- возможен процесс анализа систем с неоднородными факторами.

Учитывая вышеуказанные моменты и преимущества, в статье будет использоваться метод диагностики, базирующийся на использовании нейронной сети.

Традиционно использующимися для исследования изменений во времени спектра огибающей являются динамические искусственные нейронные сети (с задержкой во времени):

- динамическая, рекуррентная двухслойная сеть, построенная на базе MLP, нейросеть Джордона (JNN) [9, 10];

рекуррентная двухслойная сеть, построенная на базе MLP, нейросеть Элмана (ENN) / простая рекуррентная сеть (SRN) $[11,12]$;

- динамическая,

рекуррентная многослойная сеть, построенная на базе MLP, - рекуррентный многослойный персептрон (RMLP) [13, 14];

- динамическая, нерекуррентная многослойная сеть, построенная на базе MLP, - нейронная сеть с временной задержкой (TDNN) $[15,16]$.

В таблице 1 показаны характеристики динамических сетей.

\[ \begin{array}{l}\text { Таблица } 1 \\ \text { экспериментального }\end{array} \]
нейронных сетей

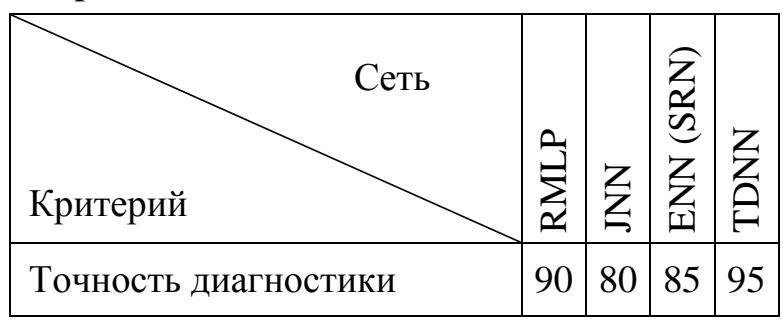

Для решения задач квалификации выбирается сеть TDNN, так как она обеспечивает наилучшую и наибольшую диагностическую точность. Пример структуры TDNN представлен на рисунке 1. 


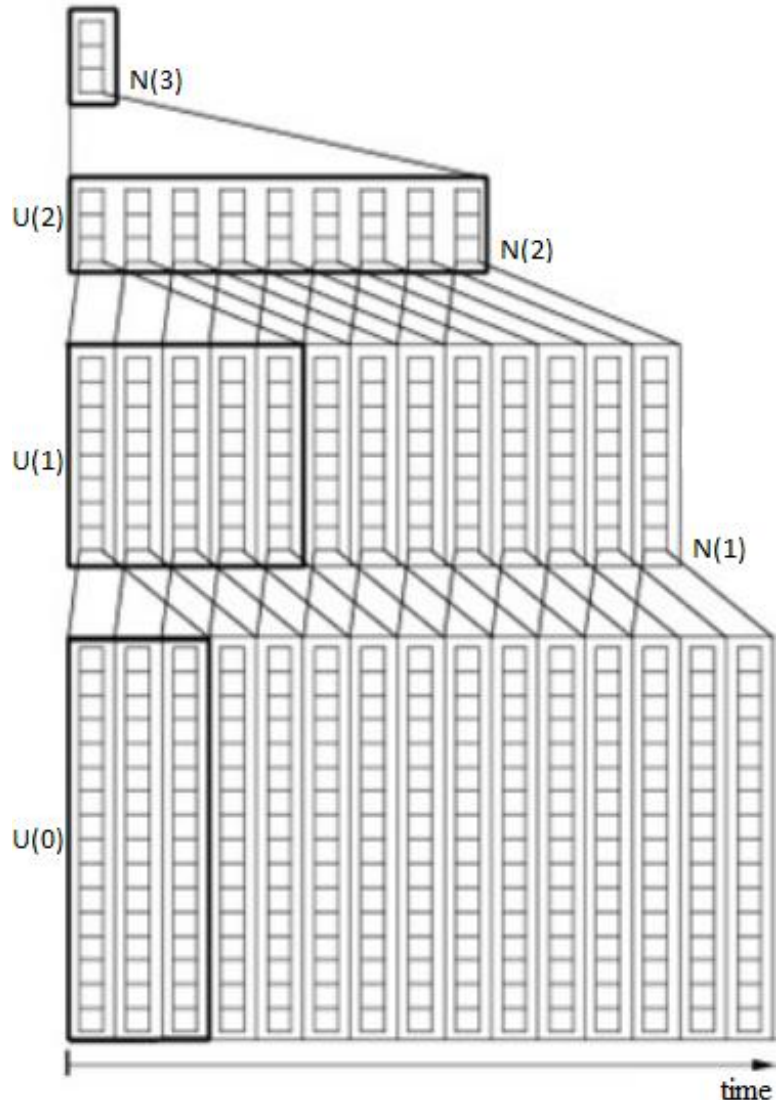

Рисунок 1 - Нейронная сеть с временной задержкой (TDNN)

Входной слой представляет собой набор модулей, каждый из которых содержит спектр огибающей в определенный момент времени.

Два скрытых слоя также представляют собой наборы модулей.

Выходной слой содержит нейроны, соответствующие классу состояния ВУГП.

Представление модели TDNN:

$$
\begin{gathered}
\mathrm{y}^{(0)}=\mathrm{x}, \\
\mathrm{y}_{\mathrm{ml}}^{(1)}=\mathrm{f}^{(1)}\left(\mathrm{b}_{\mathrm{iml}}^{(1)}+\sum_{\mathrm{i}=1}^{1+\mathrm{M}^{(0)}} \sum_{\mathrm{j}=1}^{\mathrm{N}^{(0)}} \mathrm{w}_{\mathrm{ijm} \mathrm{l}}^{(1)} \mathrm{y}_{\mathrm{ij}}^{(0)}\right),
\end{gathered}
$$

где $\mathrm{m} \in \overline{1, \mathrm{U}^{(1)}}, 1 \in \overline{1, \mathrm{~N}^{(1)}}$.

$$
\mathrm{y}_{\mathrm{ml}}^{(2)}=\mathrm{f}^{(2)}\left(\mathrm{b}_{\mathrm{jml}}^{(1)}+\sum_{\mathrm{j}=\mathrm{l}}^{1+\mathrm{M}^{(1)}} \sum_{\mathrm{i}=1}^{\mathrm{U}^{(1)}} \mathrm{w}_{\mathrm{ijml}}^{(2)} \mathrm{y}_{\mathrm{ij}}^{(1)}\right),
$$

где $\mathrm{m} \in \overline{1, \mathrm{U}^{(2)}}, 1 \in \overline{1, \mathrm{~N}^{(2)}}$.

$$
\mathrm{y}_{1 \mathrm{i}}^{(3)}=\mathrm{f}^{(3)}\left(\mathrm{b}_{\mathrm{i} 1 \mathrm{i}}^{(1)}+\sum_{\mathrm{j}=1}^{\mathrm{N}^{(2)}} \mathrm{w}_{\mathrm{ij} 1 \mathrm{i}}^{(3)} \mathrm{y}_{\mathrm{ij}}^{(2)}\right),
$$

где $\mathrm{i} \in \overline{1, \mathrm{~N}^{(3)}}$,
$\mathrm{N}^{(\mathrm{k})}$ - количество нейронов в k-м слое,

$\mathrm{w}_{\mathrm{ijml}}^{(\mathrm{k})}-$ вес связи от j-го нейрона i-го модуля к 1-му нейрону m-го модуля на k-м слое,

$\mathrm{y}_{\mathrm{ml}}^{(\mathrm{k})}$ - выход j-го нейрона m-го модуля на k-м слое,

$\mathrm{f}^{(\mathrm{k})}$ - активационная функция нейронов k-го слоя,

$$
\begin{aligned}
& \mathrm{M}^{(\mathrm{k})} \text { - задержка в k-м слое, } \\
& \mathrm{U}^{(\mathrm{k})} \text { - число модулей в k-м слое. }
\end{aligned}
$$

При этом выполняются равенства:

$$
\begin{aligned}
\mathrm{N}^{(1)}= & \mathrm{U}^{(0)}-\mathrm{M}^{(0)}, \\
\mathrm{N}^{(2)}= & \mathrm{U}^{(0)}-\left(\mathrm{M}^{(0)}+\mathrm{M}^{(1)}\right), \\
& \mathrm{U}^{(2)}=\mathrm{N}^{(3)} .
\end{aligned}
$$

На основе численных результатов работ $[15,16]$ авторами данной статьи установлено следующее соотношение, характеризующее некую зависимость:

$$
\mathrm{U}^{(0)}=2 \cdot \mathrm{U}^{(1)} .
$$

Для определения структуры модели TDNN, т. е. определения числа модулей во входном слое (число модулей в скрытых слоях вычисляется из равенств (6)-(7)), был проведен ряд экспериментов.

Результаты

обозначенных экспериментов представлены на рисунке 2 . По гистограмме, которая изображена на рисунке 2, можно отследить зависимость полученных значений среднеквадратичной ошибки от количества модулей во входном слое.

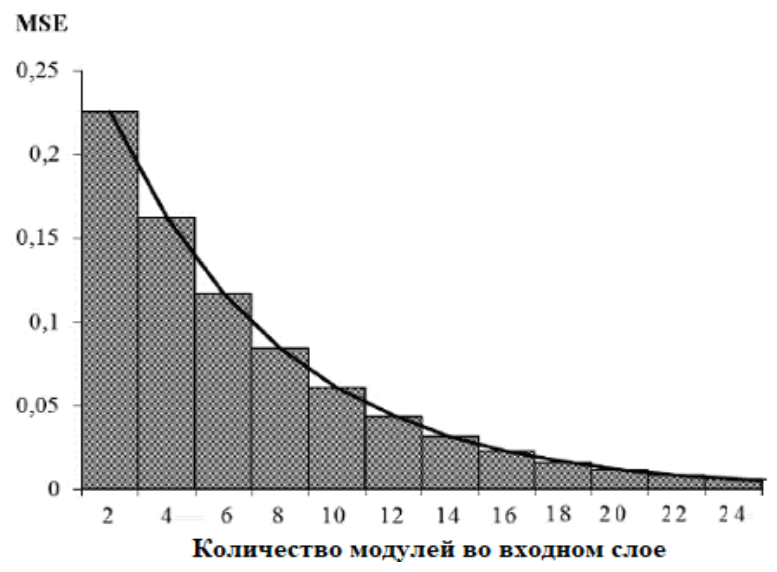

Рисунок 2 - Гистограмма, показывающая зависимости среднеквадратичной ошибки от числа модулей во входном слое 
Спектр огибающей в определенные моменты времени был использован в качестве входных данных для обучения TDNN.

Минимальный показатель (значение) среднеквадратической ошибки диагностики стал критерием выбора структуры модели сети.

Как видно из приведенного выше рисунка 2, прослеживается определенная закономерность: увеличение количества модулей входного слоя сопровождается уменьшением значения ошибки.

Для диагностики состояния ВУГП достаточно использовать 16 модулей на входном слое. Размер заявленного числа модулей обоснован тем, что по мере дальнейшего увеличения количества модулей входного слоя будет выявляться незначительное изменение значения полученной ошибки.

В работе, связанной с обучением модели TDNN, был выбран критерий, характеризующий адекватность модели. Он предусматривает выбор таких значений параметров $\mathrm{W}=\left\{\mathrm{w}_{\mathrm{ijml}}^{(1)}, \mathrm{W}_{\mathrm{ijml}}^{(2)}, \mathrm{W}_{\mathrm{ij} 11}^{(3)}\right\}$, которые обеспечивают минимизацию показателя среднеквадратичной ошибки (разницу между выходом модели и желаемым выходом):

$$
\mathrm{F}=\frac{1}{\mathrm{P}} \sum_{\mathrm{p}=1}^{\mathrm{P}}\left(\mathrm{y}_{\mathrm{p}}-\mathrm{d}_{\mathrm{p}}\right)^{2} \rightarrow \min _{\mathrm{W}}
$$

Обучение модели TDNN подчиняется критерию (8), для чего в статье приведена пакетная версия алгоритма обратного распространения (BР).

Обучение искусственной нейронной сети (ИНС), алгоритм обратного распространения:

1. Номер итерации обучения $\mathrm{n}=1$, инициализация посредством равномерного распределения на интервале $(0,1)$ или $[-0.5$, $0.5]$ порогов $b_{\mathrm{iml}}^{(1)}(\mathrm{n}), \mathrm{b}_{\mathrm{jml}}^{(2)}(\mathrm{n}), \mathrm{b}_{\mathrm{ili}}^{(3)}(\mathrm{n})$ и весов $\mathrm{w}_{\mathrm{ijml}}^{(\mathrm{k})}(\mathrm{n}), \mathrm{i} \in \overline{1, \mathrm{U}^{(\mathrm{k})}}, \mathrm{j} \in \overline{1, \mathrm{~N}^{(\mathrm{k})}}, \mathrm{m} \in \overline{1, \mathrm{U}^{(\mathrm{k}+1)}}$, $l \in \overline{1, \mathrm{~N}^{(\mathrm{k}+1)}}, \mathrm{k} \in \overline{1,3}$, где $\mathrm{N}^{(\mathrm{k})}$ - число нейронов в одном модуле $\mathrm{k}$-го слоя, $\mathrm{U}^{(\mathrm{k})}$ - число модулей $\mathrm{k}$-го слоя.

2. Задается множество для обучения $\left\{\left(\mathrm{x}_{\mu}, \mathrm{d}_{\mu}\right) \mid \mathrm{x}_{\mu} \in \mathrm{R}^{\mathrm{U}^{(0)} \times \mathrm{N}^{(0)}}, \mathrm{d}_{\mu} \in\{0,1\}^{\mathrm{N}^{(3)}}\right\}, \quad \mu \in \overline{1, \mathrm{P}}$, где $\mathrm{x}_{\mu}-\mu$-я входная обучающая матрица;

(C) Е. Е. Федоров, И. В. Ярош, Т. А. Черняк, 2019 DOI: $10.24025 / 2306-4412.4 .2019 .184525$ $\mathrm{d}_{\mu}-\mu$-й выходной обучающий вектор;

$\mathrm{N}^{(0)}$ - число нейронов в одном модуле входного слоя;

$\mathrm{U}^{(0)} \quad-$ число модулей (входных обучающих векторов);

$\mathrm{N}^{(3)}$ - число нейронов выходного слоя;

$\mathrm{P}$ - мощность обучающего множества.

3. Определение выходного сигнала $\mathrm{k}$-го слоя (прямой ход):

$$
\begin{aligned}
& \mathrm{y}_{\mu}^{(0)}(\mathrm{n})=\mathrm{x}_{\mu}, \mu \in \overline{1, \mathrm{P}}, \\
& \mathrm{y}_{\mu \mathrm{ml}}^{(1)}(\mathrm{n})=\mathrm{f}^{(1)}\left(\mathrm{s}_{\mu \mathrm{ml}}^{(1)}(\mathrm{n})\right) \text {, } \\
& \mathrm{s}_{\mu \mathrm{ml}}^{(1)}(\mathrm{n})=\sum_{\mathrm{i}=1}^{1+\mathrm{M}^{(0)}} \sum_{\mathrm{j}=0}^{\mathrm{N}^{(0)}} \mathrm{w}_{\mathrm{ijml}}^{(1)}(\mathrm{n}) \mathrm{y}_{\mu \mathrm{j}}^{(0)}(\mathrm{n}) \text {, } \\
& \mathrm{m} \in \overline{1, \mathrm{U}^{(1)}}, 1 \in \overline{1, \mathrm{~N}^{(1)}} \text {, } \\
& \mu \in \overline{1, P} \text {, } \\
& \mathrm{y}_{\mu \mathrm{ml}}^{(2)}(\mathrm{n})=\mathrm{f}^{(2)}\left(\mathrm{s}_{\mu \mathrm{m} l}^{(2)}(\mathrm{n})\right) \text {, } \\
& \mathrm{s}_{\mu \mathrm{ml}}^{(2)}(\mathrm{n})=\sum_{\mathrm{j}=1}^{1+\mathrm{M}^{(1)}} \sum_{\mathrm{i}=0}^{\mathrm{U}^{(1)}} \mathrm{w}_{\mathrm{ij} \mathrm{ml}}^{(2)}(\mathrm{n}) \mathrm{y}_{\mu \mathrm{ij}}^{(1)}(\mathrm{n}) \text {, } \\
& \mathrm{m} \in \overline{1, \mathrm{U}^{(2)}}, 1 \in \overline{1, \mathrm{~N}^{(2)}}, \\
& \mu \in \overline{1, P} \text {, } \\
& \mathrm{y}_{\mu 1 \mathrm{i}}^{(3)}(\mathrm{n})=\mathrm{f}^{(3)}\left(\mathrm{s}_{\mu 1 \mathrm{i}}^{(3)}(\mathrm{n})\right) \text {, } \\
& s_{\mu 1 \mathrm{i}}^{(3)}(n)=\sum_{\mathrm{j}=0}^{\mathrm{N}^{(2)}} \mathrm{w}_{\mathrm{ij} 1 \mathrm{i}}^{(3)}(\mathrm{n}) \mathrm{y}_{\mu \mathrm{ji}}^{(2)}(\mathrm{n}), \\
& \mathrm{i} \in \overline{1, \mathrm{~N}^{(3)}}, \mu \in \overline{1, \mathrm{P}},
\end{aligned}
$$

где $\mathrm{N}^{(\mathrm{k})}$ - количество нейронов в $\mathrm{k}-\mathrm{м}$ слое; $\mathrm{k}$ - номер слоя; $\mathrm{w}_{\mathrm{ijml}}^{(\mathrm{k})}(\mathrm{n})$ - вес связи от $\mathrm{j}$-го нейрона i-го модуля к 1-му нейрону m-го модуля на k-м слое в момент времени $\mathrm{n}$; $\mathrm{y}_{\mathrm{ml}}^{(\mathrm{k})}(\mathrm{n})$ - выход j-го нейрона m-го модуля на $\mathrm{k}-\mathrm{M}$ слое в момент времени $\mathrm{n} ; \mathrm{f}^{(\mathrm{k})}$ активационная функция нейронов k-го слоя, $\mathbf{M}^{(\mathrm{k})}$ - задержка в $\mathrm{k}$-м слое.

Считается, что:

$$
\begin{gathered}
\mathrm{w}_{\mathrm{i} 0 \mathrm{ml}}^{(1)}(\mathrm{n})=\mathrm{b}_{\mathrm{iml}}^{(1)}(\mathrm{n}), \\
\mathrm{y}_{\mathrm{i} 0}^{(1)}(\mathrm{n})=1, \\
\mathrm{w}_{0 \mathrm{jml}}^{(2)}(\mathrm{n})=\mathrm{b}_{\mathrm{jml}}^{(2)}(\mathrm{n}), \\
\mathrm{y}_{0 \mathrm{j}}^{(1)}(\mathrm{n})=1,
\end{gathered}
$$




$$
\begin{gathered}
\mathrm{w}_{\mathrm{i} 01 \mathrm{i}}^{(3)}(\mathrm{n})=\mathrm{b}_{\mathrm{i} 1 \mathrm{i}}^{(3)}(\mathrm{n}), \\
\mathrm{y}_{\mathrm{i} 0}^{(1)}(\mathrm{n})=1 .
\end{gathered}
$$

4. Расчет величины показателя энергии ошибки ИНС:

$$
\begin{gathered}
E(n)=\frac{1}{2 P} \sum_{\mu=1}^{P} \sum_{j=1}^{N^{(3)}} e_{\mu j}^{2}(n), \\
e_{\mu j}(n)=y_{\mu j}^{(3)}(n)-d_{\mu j} .
\end{gathered}
$$

5. Настройка на основе обобщенного дельта-правила синаптических весов (обратный ход):

$$
\begin{aligned}
& \frac{\partial E(n)}{\partial w_{i j 1 i}^{(3)}(n)}=\frac{1}{P} \sum_{\mu=1}^{P} y_{\mu i j}^{(2)}(n) g_{\mu 1 i}^{(3)}(n), \\
& i \in \overline{1, \mathrm{~N}^{(3)}} \text {, } \\
& \mathrm{j} \in \overline{0, \mathrm{~N}^{(2)}} \text {, } \\
& \frac{\partial \mathrm{E}(\mathrm{n})}{\partial \mathrm{w}_{\mathrm{ijml}}^{(2)}(\mathrm{n})}=\frac{1}{\mathrm{P}} \sum_{\mu=1}^{\mathrm{P}} \mathrm{y}_{\mu \mathrm{ji}}^{(1)}(\mathrm{n}) \mathrm{g}_{\mu \mathrm{ml}}^{(2)}(\mathrm{n}), \\
& \mathrm{i} \in \overline{0, \mathrm{U}^{(1)}}, \\
& \mathrm{j} \in \overline{1,1+\mathrm{M}^{(1)}} \text {, } \\
& \mathrm{m} \in \overline{1, \mathrm{U}^{(2)}}, 1 \in \overline{1, \mathrm{~N}^{(2)}}, \\
& \frac{\partial \mathrm{E}(\mathrm{n})}{\partial \mathrm{w}_{\mathrm{ijml}}^{(1)}(\mathrm{n})}=\frac{1}{\mathrm{P}} \sum_{\mu=1}^{\mathrm{P}} \mathrm{y}_{\mu \mathrm{j}}^{(0)}(\mathrm{n}) \mathrm{g}_{\mu \mathrm{ml}}^{(1)}(\mathrm{n}), \\
& \mathrm{i} \in \overline{1,1+\mathrm{M}^{(1)}}, \mathrm{j} \in \overline{0, \mathrm{~N}^{(0)}}, \\
& \mathrm{m} \in \overline{1, \mathrm{U}^{(1)}}, 1 \in \overline{1, \mathrm{~N}^{(1)}}, \\
& \mathrm{g}_{\mu 1 \mathrm{i}}^{(3)}(\mathrm{n})=\mathrm{f}^{\prime(3)}\left(\mathrm{s}_{\mu 1 \mathrm{i}}^{(3)}(\mathrm{n})\right)\left(\mathrm{y}_{\mu 1 \mathrm{i}}^{(3)}(\mathrm{n})-\mathrm{d}_{\mu \mathrm{i}}\right) \text {, } \\
& \mathrm{g}_{\mu \mathrm{ml}}^{(2)}(\mathrm{n})=\mathrm{f}^{\prime(2)}\left(\mathrm{s}_{\mu \mathrm{ml}}^{(2)}(\mathrm{n})\right) \sum_{\mathrm{i}=1}^{\mathrm{N}^{(3)}} \mathrm{w}_{\mathrm{mlli}}^{(3)}(\mathrm{n}) \mathrm{g}_{\mu 1 \mathrm{i}}^{(3)}(\mathrm{n}) \text {, } \\
& \mathrm{g}_{\mu \mathrm{ml}}^{(1)}(\mathrm{n})=\mathrm{f}^{\prime(1)}\left(\mathrm{s}_{\mu \mathrm{ml}}^{(1)}(\mathrm{n})\right) \sum_{\mathrm{i}=1}^{\mathrm{U}^{(2)}} \sum_{\mathrm{j}=\max \left\{1,1-\mathrm{M}^{(1)}\right\}}^{\min \left\{\mathrm{N}^{(2)}, 1\right\}} \mathrm{w}_{\mathrm{mij}}^{(2)}(\mathrm{n}) \times \\
& \times \mathrm{g}_{\mu \mathrm{ij}}^{(2)}(\mathrm{n}) \text {. }
\end{aligned}
$$

6. Проверка условия завершения.

Если $\mathrm{E}(\mathrm{n})<\varepsilon$, то работа алгоритма завершится, иначе $\mathrm{n}=\mathrm{n}+1$ и переход к 2 .

Использование пакетного режима обучения для нейросети TDNN на видеокарте GeForce $920 \mathrm{M}$ позволило произвести ускорение прямого хода примерно в $\mathrm{P}\left(\mathrm{U}^{(1)} \times \mathrm{N}^{(1)}+\mathrm{U}^{(2)} \times \mathrm{N}^{(2)}+\mathrm{N}^{(3)}\right)$ ра3, а обратного хода - в количество раз, рассчитываемое как $\frac{\mathrm{P}}{\log _{2} \mathrm{P}}\left(\mathrm{U}^{(0)} \times \mathrm{N}^{(0)} \times \mathrm{U}^{(1)} \times \mathrm{N}^{(1)}+\mathrm{U}^{(2)} \times \mathrm{N}^{(2)} \times \mathrm{U}^{(1)} \times \mathrm{N}^{(1)}+\mathrm{N}^{(2)} \times \mathrm{N}^{(3)}\right)$,

(C) Е. Е. Федоров, И. В. Ярош, Т. А. Черняк, 2019 DOI: $10.24025 / 2306-4412.4 .2019 .184525$ при этом использовалась редукция для осуществления обратного хода при сложении по обучающему множеству.

Выводы. Для решения проблемной ситуации, связанной с повышением показателя необходимости осуществления диагностики состояния ВУГП, были исследованы соответствующие методы. Результаты выполненных исследований показывают, что наиболее эффективным для диагностики является использование искусственных нейронных сетей.

Для повышения показателей качества результатов диагностики была выбрана нейронная сеть TDNN, a также была определена структура ее модели.

Численные исследования и выполненные эксперименты показали, что при наличии 16 и более модулей во входном слое значение стандартной ошибки существенно не изменяется, а выбранная нейронная сеть дает диагностический результат с наименьшим (минимальным) отклонением.

Использование предложенного в данной работе пакетного режима нейросетевого обучения позволило произвести ускорение прямого хода примерно в $\mathrm{P}\left(\mathrm{U}^{(1)} \times \mathrm{N}^{(1)}+\mathrm{U}^{(2)} \times \mathrm{N}^{(2)}+\mathrm{N}^{(3)}\right)$ раз, а ускорение обратного хода - примерно в $\frac{\mathrm{P}}{\log _{2} \mathrm{P}}\left(\mathrm{U}^{(0)} \times \mathrm{N}^{(0)} \times \mathrm{U}^{(1)} \times \mathrm{N}^{(1)}+\mathrm{U}^{(2)} \times \mathrm{N}^{(2)} \times \mathrm{U}^{(1)} \times \mathrm{N}^{(1)}+\mathrm{N}^{(2)} \times \mathrm{N}^{(3)}\right)$, pa3.

Практическая значимость выражена в возможности увеличения скорости прямого и обратного хода при использовании представленного пакетного режима обучения нейронной сети. Предложенный подход может быть использован и применен в различных интеллектуальных системах, обеспечивающих диагностические процессы.

\section{Перспективы}

дальнейших исследований связаны с осуществлением последующей работы, направленной на разработку иных методик углубленного изучения процесса изменения состояния ВУГП и на исследование возможных интеллектуальных методов диагностики ВУГП.

\section{Список литературы}

[1] А. Р. Ширман, и А. Б. Соловьев, Практическая вибродиагностика $и$ мониторинг состояния механического оборудования. Москва, 1996. 
[2] А. В. Барков, Н. А. Баркова, и А. Ю. Азовцев, Мониторинг и диагностика роторных машин по вибращии. СанктПетербург: Изд. центр СПбГМТУ, 2000.

[3] В. А. Барков, Современное состояние виброакустической диагностики машин. Санкт-Петербург: Ассоциация ВАСТ, 2002.

[4] А. С. Гольдин, Вибрациия роторных машин. Москва: Машиностроение, 1999.

[5] В.В.Клюева, Неразрушающий контроль u диагностика. Москва: Машиностроение, 2003.

[6] Е. Е. Федоров, Методики интеллектуальной диагностики. Донецк: Ноулидж, 2010.

[7] Г. А. Бабак, $\quad$ К. П. Бочаров, и А. Т. Волохов, Шахтные вентиляторные установки главного проветривания. Москва: Недра, 1982.

[8] Б. А. Носырев, и С. В. Белов, Вентиляторные установки шахт $u$ метрополитенов. Екатеринбург: Изд-во Урал. гос. горно-геолог. акад., 2000.

[9] M. I. Jordan, "Attractor dynamics and parallelism in a connectionist sequential machine", in Proc. Ninth Annu. Conf. Cognitive Science Society, Hillsdale, NJ, 1986, pp. 531-546.

[10] M. Jordan, and D. Rumelhart, "Forward models: supervised learning with a distal teacher", Cognitive Science, vol. 16, pp. 307-354, 1992.

[11] Z. Zhang, Z. Tang, and C. Vairappan, "A novel learning method for Elman neural network using local search", Neural Information Processing - Letters and Reviews, vol. 11, no. 8, pp. 181-188, 2007.

[12] J. Wiles, and J. Elman, "Learning to count without a counter: a case study of dynamics and activation landscapes in recurrent networks", in Proc. Seventeenth Annu. Conf. Cognitive Science Society, Cambridge, MA, 1995, pp. 1200-1205.

[13] S. Haykin, Neural networks. NY: Pearson Education, 1999.

[14] Е. Е. Федоров, Искусственные нейронные сети. Красноармейск: ДонНТУ, 2016.

[15] K. J. Lang, and G. E. Hinton, "The development of the time-delay neural network architecture for speech recognition", Pittsburgh, PA, CarnegieMellon University, Tech. Rep. CMU-CS-88152-1988.

[16] A. Waibel, T. Hanazawa, G. Hinton, K. Shikano, and K. J. Lang, "Phoneme recognition using time-delay neural networks", IEEE transactions on Acoustics, Speech and Signal Processing, vol. 37, pp. 328-329, 1988.

\section{References}

[1] A. R. Shirman, and A. B. Solov'ev, Practical vibration diagnostics and monitoring of mechanical equipment condition. Moscow, 1996 [in Russian].

[2] A. V. Barkov, N. A. Barkova, and A. Yu. Azovczev, Monitoring and diagnostics of rotary machines by vibration, St. Petersburg: Izd. czentr SPbGMTU, 2000 [in Russian].

[3] V. A. Barkov, Current state of vibroacoustic diagnostics of machines. St. Petersburg: Assotsiatsiya VAST, 2002 [in Russian].

[4] A. S. Gol'din, Vibration of rotary machines. Moscow: Mashinostroenie, 1999 [in Russian].

[5] V. V. Klyueva, Non-destructive testing and diagnostics. Moscow: Mashinostroenie, 2003 [in Russian].

[6] E. E. Fedorov, Methods of intellectual diagnostics. Donetsk: Noulidzh, 2010 [in Russian].

[7] G. A. Babak, K. P. Bocharov, and A. T. Volokhov, Mine fan installations of main ventilation. Moscow: Nedra, 1982 [in Russian].

[8] B. A. Nosyrev, and S. V. Belov, Fan installations of mines and undergrounds. Ekaterinburg: Izd-vo Ural. gos. gornogeolog. akademii, 2000 [in Russian].

[9] M. I. Jordan, "Attractor dynamics and parallelism in a connectionist sequential machine", in Proc. Ninth Annu. Conf. Cognitive Science Society, Hillsdale, NJ, 1986, pp. 531-546.

[10] M. Jordan, and D. Rumelhart, "Forward models: supervised learning with a distal teacher", Cognitive Science, vol. 16, pp. 307-354, 1992.

[11] Z. Zhang, Z. Tang, and C. Vairappan, "A novel learning method for Elman neural network using local search", Neural Information Processing - Letters and Reviews, vol. 11, no. 8, pp. 181-188, 2007.

[12] J. Wiles, and J. Elman, "Learning to count without a counter: a case study of dynamics and activation landscapes in recurrent networks", in Proc. Seventeenth Annu. Conf. Cognitive Science Society, Cambridge, MA, 1995, pp. 1200-1205.

[13] S. Haykin, Neural networks. NY: Pearson Education, 1999. 
[14] E. E. Fedorov, Artificial Neural Networks. Krasnoarmejsk: DonNTU, 2016 [in Russian].

[15] K. J. Lang, and G. E. Hinton, "The development of the time-delay neural network architecture for speech recognition", Pittsburgh, PA, Carnegie-
Mellon University, Tech. Rep. CMU-CS-88152-1988.

[16] A. Waibel, T. Hanazawa, G. Hinton, K. Shikano, and K. J. Lang, "Phoneme recognition using time-delay neural networks", IEEE transactions on Acoustics, Speech and Signal Processing, vol. 37, pp. 328-329, 1988.

E. E. Fedorov ${ }^{1}$, I. V. Yarosh ${ }^{2}$, T. O. Cherniak ${ }^{2}$,

${ }^{1}$ Cherkasy State Technological University

Shevchenko blvd, 460, Cherkasy, 18006, Ukraine,

${ }^{2}$ Donetsk National Technical University

Shybankova Square, 2, Pokrovsk, 85300, Ukraine

\section{TDNN NEURAL NETWORK FOR DIAGNOSING THE STATE OF THE FAN INSTALLATION OF THE MAIN AIRING}

Currently, the increasing of operational safety is one of the major problems that exist in the mining industry. The problem of emergency equipment for mining industry is caused by the rapid increase in the share of depletion of its physical resources. Among the existing mine equipment, one of the most important roles is played by fan installations of the main airing, which ensure normal vital activity of the mine personnel. Therefore, an important task is to develop a software component, designed to diagnose the state of it and to be used in computer systems. At the heart of this objective lies the problem of building effective methods, providing high speed of diagnostics model training, as well as a high probability, adequacy and speed of signals recognition, which contain the vibrational information. At present, as a tool for vibration diagnostics, the following calculation methods are most commonly used: kurtosis, crest factor, RMS value, envelope spectrum. However, when using these markers separately for diagnosis of fan installations of the main airing condition, the probability of error is no less than 0.05. On the other hand, the processing speed of vibrational information is poor. Therefore, the development of methods for intelligent integrated diagnostics of fan installations of the main airing is relevant.

As the use of artificial neural networks in the diagnosis gives tangible advantages, which are the following: the interaction between the factors is studied on finished models; it does not require any assumptions regarding the distribution of factors; a priori information about the factors can be omitted; the initial data can be highly correlated, incomplete or noisy; it is possible to conduct the analysis of systems with a high degree of nonlinearity; fast model development; high adaptability; the analysis of systems with a large number of factors; it does not require a complete enumeration of all possible models; the analysis of systems with non-uniform factors, neural network method of diagnosis is used in the article. The aim of the study consists in the development of a method for analysis of the process of changing the condition of fan installations of the main airing. The article defines the structure of the artificial neural network model, which is a TDNN neural network, that allows to explore the spectrum envelope at certain points of time. The minimum root-mean-square error has been chosen as a criterion for evaluating the effectiveness of the neural network diagnostic model. As a result of a numerical study, it is found that in the presence of 16 modules in the input layer, the value of RMS error does not change significantly, the proposed network provides diagnostic results with a minimum deviation. The use of the proposed batch training mode has made it possible to accelerate the forward stroke for about $\mathrm{P}\left(\mathrm{U}^{(1)} \times \mathrm{N}^{(1)}+\mathrm{U}^{(2)} \times \mathrm{N}^{(2)}+\mathrm{N}^{(3)}\right)$ times, and the reverse stroke - for about $\frac{\mathrm{P}}{\log _{2} \mathrm{P}}\left(\mathrm{U}^{(0)} \times \mathrm{N}^{(0)} \times \mathrm{U}^{(1)} \times \mathrm{N}^{(1)}+\mathrm{U}^{(2)} \times \mathrm{N}^{(2)} \times \mathrm{U}^{(1)} \times \mathrm{N}^{(1)}+\mathrm{N}^{(2)} \times \mathrm{N}^{(3)}\right)$, times, where $P$ is the power of the training set, $N^{(k)}$ is the number of neurons in k-layer, $U^{(k)}$ is the number of modules in the k-layer. The created algorithms can be used for solving problems related to the diagnostics of electromechanical objects.

Keywords: diagnostics, fan installation of the main airing, neural network, TDNN, operational safety, batch training mode.

Стаття надійшла 05.11.2019

Прийнято 29.11.2019

(c) Е. Е. Федоров, И. В. Ярош, Т. А. Черняк, 2019 DOI: $10.24025 / 2306-4412.4 .2019 .184525$ 